\title{
Excessive production of ascitic fluid after laparoscopic operation for early-stage ovarian cancer
}

\author{
Ioannis Kotsopoulos • Dimitrios Evaggelinos • \\ Paraskevi Skafida • Vasilios Kartsiounis • \\ Nektarios Chalvatzas • Andreas Kavallaris
}

Received: 5 June 2010 /Accepted: 12 July 2010 /Published online: 23 July 2010

(C) Springer-Verlag 2010

Keywords Clear cell carcinoma - Ovarian cancer .

Laparoscopy · Ascitic fluid · Adhesiolysis .

Pseudo-membrane

\section{Background}

Peritoneal implants and/or venous or lymphatic obstruction, presenting in advanced stages of ovarian cancer, stimulate production of ascitic fluid [1]. Also, postoperative production of ascitic fluid after operations for advanced ovarian cancer is not rare. Especially in end-stage ovarian cancer, symptomatic and rapidly reaccumulating ascitic fluid usually needs repeated paracenteses or drainage though peritoneal catheter. In addition, thoracentesis, pleurodesis, or catheter has already been used to drain pleural fluid [2]. On the other hand, in early-stage (Ia) ovarian cancer, considering the absence of implants, postoperative ascites usually cannot be cancer-related. In this paper, a rare complication of excessive production of ascitic fluid after laparoscopic operation for early-stage clear cell ovarian carcinoma and the used of treatment method are presented.

I. Kotsopoulos $\cdot$ D. Evaggelinos $\cdot$ P. Skafida $\cdot$ V. Kartsiounis Gynecologic Oncology Department,

"Theagenio" Anticancer Hospital of Thessaloniki,

Thessaloniki, Greece

N. Chalvatzas $\cdot$ A. Kavallaris $(\square)$

Department of Gynecology and Obstetrics,

University of Schleswig-Holstein, Campus Luebeck,

Luebeck, Germany

e-mail: andreas.kavallaris@uk-sh.de

\begin{abstract}
Methods
In this paper, authors present a case of a 27-year-old Greek woman, gravid 0 , with history of early-stage clear cell ovarian carcinoma diagnosed through histopathological examination of a $16 \times 18-\mathrm{cm}$ cystic tumor from the left ovary. Patient's medical history includes hypothyroidism well controlled with levothyroxine sodium tabs and no history of allergy. CT control indicated no residual or recurrent disease. Although clear cell carcinoma is more likely to be diagnosed at early stage, this type is associated with pooper prognosis compared to serous cancers [3]. So, a second look operation was decided due to histological type of the tumor. The patient underwent laparoscopic hysterectomy, bilateral oophorosalpingectomy, omentectomy, bilateral external ilium lymphadenectomy, using ultrasound knife, and staging.
\end{abstract}

\section{Findings}

The deduction of the pathological and cytological examination indicated no residual malignancy (FIGO stage Ia). During the operation, there was thermal injury of the obturator nerve, probably caused by the use of ultrasound knife and surgeons' fatigue (prolonged operation, $9 \mathrm{~h}$, more than 4201 of $\mathrm{CO}_{2}$ were used with a pressure of $18 \mathrm{mmHg}$ ). Suturing of the nerve through laparotomy was immediately operated. Postoperative motility disorders, as femoral internal rotation disorder and adduction incapacity, were totally restored in 10 days. However, there was an excessive production of ascitic fluid and edema of the legs, starting from postoperative day 5. Fluid's cytological and 
microbiologic controls, including chlamydia, were negative and biochemical examination indicated transudate. CT and MRI revealed both ascitic and pleural fluid (causing respiratory distress). Ultrasound of hepatic veins and inferior vena cava and MRI of ilium veins were normal. HIV, HBV, HCV, TOXO, EBV, and CMV examination were also negative. Until postoperative day 53, about 211 of ascitic fluid was drained and no applied treatment was able to control the production.

A second laparoscopy was decided performing by specialist in laparoscopic oncological surgery. During the operation, a pseudo-membrane covering entire peritoneal cavity was found and extracted (Figs. 1, 2, and 3). The pseudo-membrane covered the whole abdomen and was filled with ascitic fluid, this kind of cyst pressed on the hepatic vessels. Also, ascitic fluid was drained and adhesiolysis with partial resection of the pseudo-membrane was operated (Fig. 3). Patient was discharged home on postoperative day 7 without complications and with no more ascitic or pleural fluid production. Authors consider that pressure from the pseudo-membrane to liver vessels was the main factor causing this rare complication. On the other hand, possible infection could also be accused, but the use of antibiotics combination caused no indication of infection in fluid analysis.

\section{Discussion and conclusion}

Laparoscopic management of ovarian cancer [4,5] and especially laparoscopic omentectomy are feasible. Our experience indicated that these procedures should be performed by specialist in laparoscopic oncological surgery with experience in advanced laparoscopy. In addition,

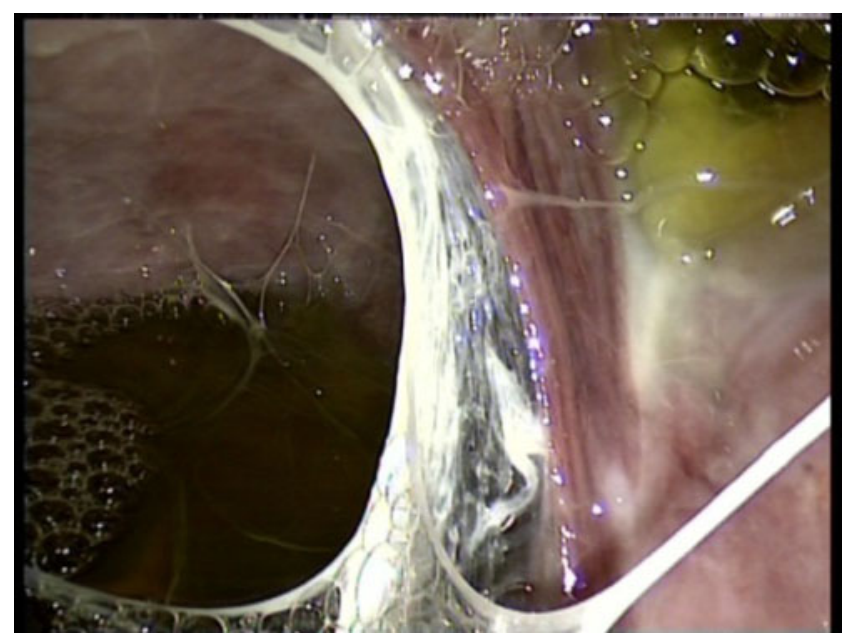

Fig. 2 Pseudo-membrane, ascitic fluid, and adhesions

although the use of ultrasound knife can improve laparoscopic operation, there is still danger of thermal injuries.

In early-stage ovarian cancer, considering the absence of implants and obstruction of veins or lymphatic vessels, there is no production of pre- or postoperative ascitic fluid. Moreover, taking in consideration that not only cancer but also infections and other diseases can produce excessive postoperative ascitic fluid, control must include cytological and microbiological examination of the fluid, virus' antibodies examination of blood serum, and imaging methods. Finally, formation of pseudo-membranes and excessive production of ascitic fluid due to compression of hepatic vessels from these membranes or adhesions could be very rare complications after operations for early-stage ovarian cancer. Adhesiolysis and ascites drainage seem to be an indicated treatment procedure.

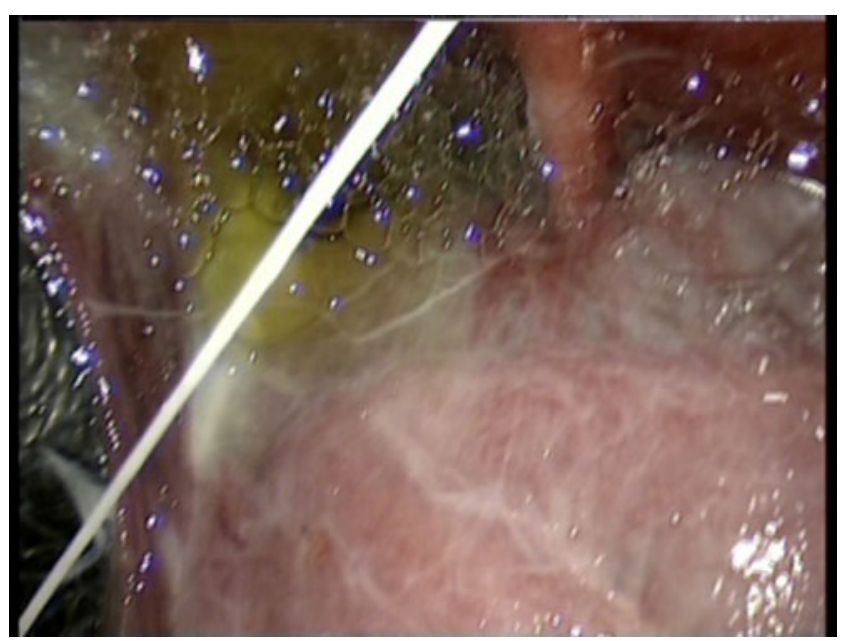

Fig. 1 Pseudo-membrane covering liver

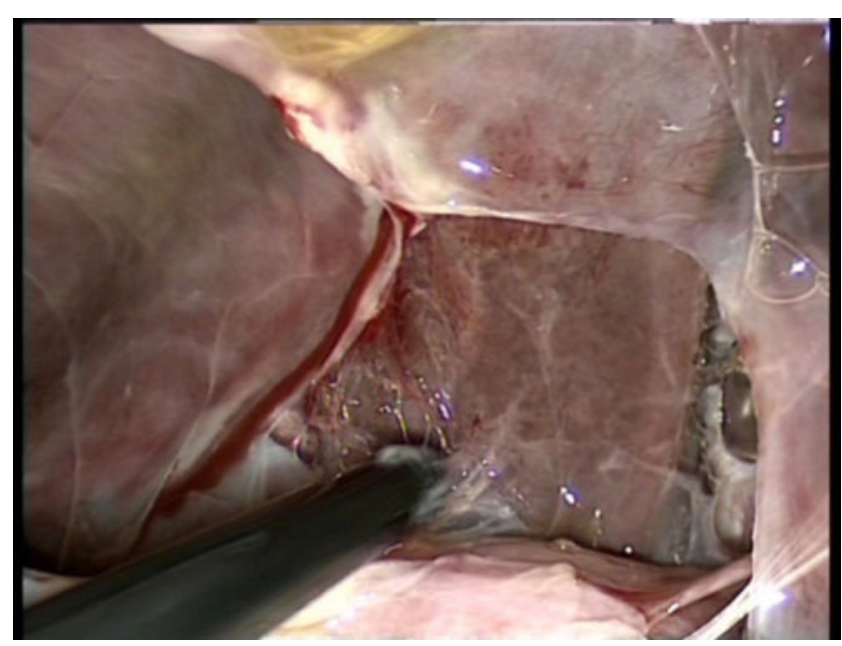

Fig. 3 Laparoscopic lysis of the pseudo-membrane and adhesiolysis 
Conflicts of interest The authors report no conflicts of interest. The authors alone are responsible for the content and writing of the paper.

\section{References}

1. Doherty GM (2006) Current surgical diagnosis \& treatment. International Twelfth edition, vol 22. McGraw-Hill, New York, p 504

2. Schorge J, Schaffer J, Halvorson L, Hoffman B, Brandshaw K, Cunningham FG (2008) Williams gynecology, vol 35. McGrawHill, New York, p 733
3. Chan JK, Teoh D, Hu JM, Shin JY, Osann K, Kapp DS (2008) Do clear cell ovarian carcinomas have poorer prognosis compared to other epithelial cell types? A study of 1411 clear cell ovarian cancers. Gynecol Oncol 109(3):370-376

4. Nezhat FR, Ezzati M, Chuang L, Shamshirsaz AA, Rahaman J, Gretz H (2009) Laparoscopic management of early ovarian and fallopian tube cancers: surgical and survival outcome. Am J Obstet Gynecol 200(1):83.e1-83.e6

5. Jung US, Lee JH, Kyung MS, Choi JS (2009) Feasibility and efficacy of laparoscopic management of ovarian cancer. J Obstet Gynaecol Res 35(1):113-118 